\title{
Stochastic filtering approach for condition-based maintenance considering sensor degradation
}

\author{
Bin Liu, Member, IEEE, Phuc Do, Benoit Iung, and Min Xie, Fellow, IEEE
}

\begin{abstract}
This paper proposes a condition-based maintenance policy for a deteriorating system whose state is monitored by a degraded sensor. In the literature of condition-based maintenance, it is commonly assumed that inspection of system state is perfect or subject to measurement error. The health condition of the sensor, which is dedicated to inspect the system state, is completely ignored during system operation. Yet due to the varying operation environment and aging effect, the sensor itself will suffer a degradation process and its performance deteriorates with time. In presence of sensor degradation, Kalman filter is employed in this paper to progressively estimate the system and the sensor state. Since estimation of system state is subject to uncertainty, maintenance solely based on the estimated state will lead to a sub-optimal solution. Instead predictive reliability is used as a criterion for maintenance decision-making, which is able to incorporate the effect of estimation uncertainty. Preventive replacement is implemented when the estimated system reliability at inspection hits a specific threshold, which is obtained by minimizing the long run maintenance cost rate. An example of wastewater treatment plant is used to illustrate the effectiveness of the proposed maintenance policy. It can be concluded through our research that (i) disregarding the sensor degradation while it exists will significantly increase the maintenance cost; (ii) the negative impact of sensor degradation can be diminished via proper inspection and filtering methods.
\end{abstract}

Note to Practitioners-This paper was motivated by the observation of sensor degradation in wastewater treatment plants but the developed approach also applies to other systems such as manufacturing systems, chemical plants, and pharmaceutical factories, where sensors are dedicated to a long-time operation in harsh environment. This paper investigates the impact of sensor degradation on condition-based maintenance and suggests that the effect of sensor degradation should be carefully addressed while making maintenance decisions. Otherwise, it will lead to a sub-optimal maintenance decision and increase the operating cost. An optimal maintenance decision, which contains the optimal inspection interval and reliability threshold, is achieved via minimizing the long run cost rate. In presence of measurement noise and intrinsic uncertainty from degradation, a stochastic filtering approach is employed to estimate the system and sensor state. Based on the estimated states and the calculated reliability, a dynamic maintenance decision is obtained at each inspection. This study can be further extended considering non-Gaussian noise and alternative degradation processes.

Index Terms-Condition-based maintenance, imperfect inspection, sensor degradation, Wiener process, stochastic filter.

B. Liu is with the Department of Management Science, University of Strathclyde, Glasgow, G1 1XQ, UK (e-mail: b.liu@ strath.ac.uk)

P. Do and B. Iung are with Lorraine University, CRAN, CNRS UMR 7039, Campus Sciences BP70239, 54506 Vandoeuvre, France (email:vanphuc.do@univ-lorraine.fr; benoit.iung@univ-lorraine.fr)

M. Xie is with the Department of Systems Engineering and Engineering Management, City University of Hong Kong, Kowloon, Hong Kong (e-mail: minxie@cityu.edu.hk)

\section{INTRODUCTION}

$\mathbf{N}$ OWADAYS, with the increasing requirement of high reliability and safety for modern systems, advanced datacollecting techniques are widely used to monitor system health condition, either continuously or periodically. The ability to gather system health information significantly prompts the development of condition-based maintenance (CBM). In the past few decades, CBM has received an increasing attention, due to its capability to intervene system performance in time. As opposite to traditional time-based maintenance policies, CBM has shown its advances in preventing system failure and reducing operating cost from both the academic and practical point of view [1]-[3].

The effectiveness of CBM policy highly depends on the appropriateness of system degradation modeling and the inspection/monitoring accuracy. In terms of the inspection accuracy, CBM can be classified into two categories: CBM with perfect inspection and with imperfect inspection [4]-[9]. The assumption of perfect information eliminates the effect of measurement noise and allows the CBM more focused on the development of optimal maintenance policy. In literature, numerous CBM models have been proposed assuming perfect inspection (e.g., [10]-[13]).

In practical applications, however, sensor is often subject to noise and perfect inspection cannot be achieved [14], [15]. For this reason, the assumption of perfect inspection is relaxed to establish a more realistic model, which leads to the development of CBM with imperfect inspection. Actually, the existing CBM policies with imperfect inspection primarily follow two streams. One stream formulates the maintenance issue into the framework of partially observable Markov decision process (POMDP) or its variants [16]-[20]. For example, [21] developed a CBM policy for a deteriorating system with partially observable environment, where the degradation rate is determined by the operating environment. POMDP model was formulated to achieve the optimal maintenance decision. [22] investigated the CBM issue for a machine subject to imperfect continuous monitoring. A continuous-observation partially observable semi-Markov decision process was presented to select various maintenance actions.

The other stream embeds a CBM policy with system state estimation and degradation parameter updating [23]-[25]. [25] developed a maintenance model with a sensor-based updating scheme, where the optimal maintenance routine was scheduled based on the updated degradation parameters and estimated remaining useful life distribution. [26] developed a maintenance model under indirect condition monitoring, where the value 
of condition monitoring and impact of measurement error are investigated in detail. [27] presented a risk sensitive particle filter for prognostic, which was further applied in maintenance scheduling. [28] proposed a CBM policy using real-time remaining useful life prediction for a multi-component system with stochastic dependence. Although imperfect monitoring is considered, an implicit assumption of the previous studies is that the sensor performance remains steady within its life cycle, implying constant measurement noise or inspection error.

However, due to the varying operating environment and cumulative damage to the equipment and the dedicated sensors, the assumption of constant inspection quality during the life cycle is increasingly challenged. In real-life application, the performance of sensor usually deteriorates over a long period of operating time [29]-[31]. [32] investigated the impact of sensor degradation on control system and developed an approach to optimally improve the system reliability. One of the most serious impairment is sensor drift, which has plagued the research community for decades. In general, sensor drift can be attributed primarily to two sources [33], [34]. One is due to the internal degradation as a result of chemical and physical interaction during the operation period. The other derives from external and uncontrollable operating environment, such as variations of temperature and humidity. Existence of sensor degradation will significantly influence the effectiveness of system health prognostic and the associated CBM policies [35], which will lead to increased maintenance and operating cost if no counteracting measures are implemented to deal with the sensor degradation.

The impact of sensor degradation lies in the distortion of measurements, which makes the observations biased and severely deviate from the true values [36]. Without proper approaches or measures to diminish the negative effect of sensor degradation, estimation of system state and the associated maintenance policy will have to be conducted based on the fraudulent information provided by the sensors. As a result, the maintenance decisions will deviate from the optimal one and maintenance actions will be ineffectively performed, which endangers the operating system and causes huge economic losses. Our case study shows that the maintenance cost without addressing the sensor degradation is much higher than the maintenance cost that has properly handled the sensor degradation, which indicates that the sensor degradation exerts a significant impact on maintenance decision.

To the best of our knowledge, no previous studies have covered the issue of CBM with sensor degradation, despite its prevalence and criticality in industrial applications. Motivated by the practical need of CBM models considering sensor degradation, in this paper, we investigate the effect of sensor degradation on CBM decision-making. A system is subject to a continuous degradation, described by Wiener process, along with a sensor to inspect the system state. However, the inspection is imperfect in the sense of measurement noise and time-varying drift as a result of sensor degradation. To face with this issue, stochastic filtering is employed to estimate the drift level and system state as a first step, followed by the CBM model. The optimal maintenance policy is achieved by minimizing the long run cost rate.

The remainder of this paper is organized as follows. Section II describes the degradation process of the system and sensor, and the measurement process. In Section III, the proposed maintenance policy is firstly presented. Then, a maintenance cost model is formulated based on the imperfect observation. An initial guess based on perfect inspection is herein proposed to serve for optimization algorithm. Section IV describes the estimation process and impact of sensor failure on maintenance action at inspection, where Kalman filter is employed to estimate the system and sensor states. In Section V, an example of wastewater treatment plant is used to illustrate the effectiveness of the proposed maintenance policy. Finally, conclusions and future research directions are provided in Section VI.

\section{SYSTEM \& SENSOR DEGRADATION PROCESS}

Consider a system subject to a continuous-time degradation process. Wiener process is employed to describe the underlying degradation progression. Wiener process exhibits a nonmonotone degradation path, which has successfully captured the degradation characteristics of many real-life systems [37], [38]. Let stochastic process $X(t), t \geq 0$ denote the associated degradation process over the operating time $t$, which is expressed as

$$
X(t)=X(0)+\lambda t+\sigma B(t)
$$

where $\lambda$ is the drift coefficient, $\sigma$ is the diffusion coefficient, and $B(t)$ is the standard Brownian motion. $X(0)$ is the initial degradation level, and $\sigma B(t) \sim N\left(0, \sigma^{2} t\right)$ stands for the randomness of the degradation process. Without loss of generality, it is assumed $X(0)=0$.

Sensor is dedicated to inspecting the system state. However, due to the varying environmental factor and cumulative damage, the dedicated sensor is subject to a degradation process. It is assumed that the degradation of sensor can be characterized by increase of drift and measurement inaccuracy, which is modeled as Wiener process, i.e.,

$$
S(t)=S(0)+\eta t+\delta B(t)
$$

where $S(t)$ is the sensor degradation level at time $t, \eta$ and $\delta$ are the drift and diffusion coefficients respectively. Note that $\eta$ can be positive or negative, denoting the positive or negative drift. It is also assumed that the system and sensor degrade independently and the degradation parameters are known in advance. Actually the parameters can be estimated with offline historical failure/degradation data. Numerous existing methods serve for the estimation purpose, e.g., maximum likelihood estimation and moment estimation [39], [40]. We do not present the parameter estimation procedure since is out of the scope of this paper.

Let $\{Y(t), t \geq 0\}$ denote the measurement process, which relates the uncertain observation with the underlying system and sensor degradation state at time $t$. Combining the influence of sensor and system degradation, the measurement at time $t$ is given as

$$
Y(t)=X(t)+S(t)+\varepsilon
$$




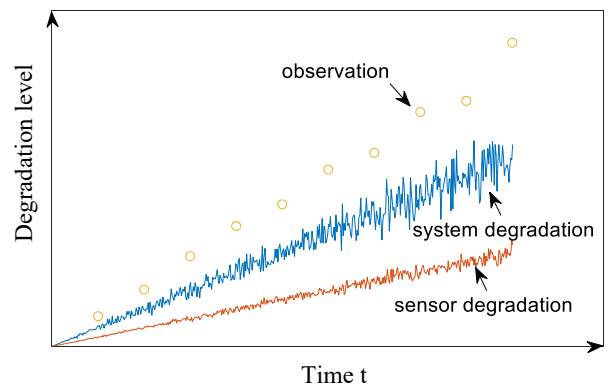

Fig. 1: Sketch of observation, system \& sensor degradation

where $\varepsilon$ is the statistically independent and identically distributed measurement error, following normal distribution $\varepsilon \sim$ $N\left(0, \gamma^{2}\right)$ at any time point.

Fig. 1 depicts the system \& sensor degradation and measurements at inspection. If there exists sensor degradation, the measurements at inspection deviate from the system degradation. If no sensor degradation is considered, the measurement at time $t$ is reduced to $Y(t)=X(t)+\varepsilon$, which is identical as the measurement process in traditional works.

Following the tradition of first-passage-time (FPT), system failure time is defined as the epoch when the system degradation level hits the pre-specified threshold $\zeta$ for the first time. System lifetime $T$ is interpreted as the FPT to the pre-specified failure threshold, i.e.,

$$
T=\inf \{t: X(t) \geq \zeta\}
$$

The probability density function (pdf) and cumulative distribution function (cdf) of system lifetime $T$ are given as [38]

$$
\begin{aligned}
f_{T}(t)= & \frac{\zeta}{\sqrt{2 \pi t^{3} \sigma^{2}}} \exp \left(-\frac{\zeta-\lambda t}{2 \sigma^{2} t}\right) \\
F_{T}(t) & =1-\Phi\left(\frac{\zeta-\lambda t}{\sigma \sqrt{t}}\right) \\
& +\exp \left(\frac{2 \lambda \zeta}{\sigma^{2}}\right) \Phi\left(\frac{-\zeta-\lambda t}{\sigma \sqrt{t}}\right)
\end{aligned}
$$

Remark 2.1: In this study we use Wiener process to illustrate our approach. Actually, sensors in different industries will exhibit different degradation processes. The sensor can be subject to various degradation processes, such as Gamma process, inverse Gaussian process, etc., depending on the application and the environmental influence. But our method can be applied as well. In addition, the error could be a linear or non-linear function with respect to the sensor degradation level. In this study, a linear form is used. It should be noted that if the system is subject to a non-linear degradation, we may need to resort to other filtering approach such as extended Kalman filter or particle filter.

\section{FRAMEWORK OF CONDITION-BASED MAINTENANCE MODEL}

In this section, we will describe the maintenance policy and formulate the associated maintenance cost model. Long-run maintenance cost rate is employed as the criterion to evaluate the proposed policy. The optimal maintenance decision is achieved via an optimization procedure with a near-optimal initial guess.

\section{A. Description of the maintenance policy}

The system under consideration is subject to discrete inspection. Let $\left\{t_{k}, k=0,1,2, \ldots\right\}$ denote the inspection time, $0=t_{0}<t_{1} \ldots<t_{k}$. Denote $y_{k}=Y\left(t_{k}\right)$ as the observation at time $t_{k}$. The set of degradation measurements is denoted $\mathbf{Y}_{1: k}=\left\{y_{1}, y_{2}, \ldots, y_{k}\right\}$. Let $x_{k}=X\left(t_{k}\right)$ represent the system degradation state at time $t_{k}$.

Based on the Kalman filter, the system state is updated when new observation arrives, which leads to a nonstationary degradation process. Therefore, we resort to a dynamic maintenance policy to effectively prevent system failure, which determines the optimal maintenance action at each inspection epoch, given the inspection history $\mathbf{Y}_{1: k}$.

It is assumed that the system failure is not self-announcing, i.e, system failure can only be detected at inspection, which is referred to as soft failure [37], [41]. Note that soft failure may not necessarily indicate physical failure (catastrophic failure), but can be the performance of a system that fails to satisfy the demands. Soft failure is commonly assumed in maintenance literatures and industrial applications [38]. Particularly, for safety-critical systems, a system is deemed failed when its safety or reliability drops to a certain level. Following the industrial practice, periodic inspection is used to monitor the system state. Let $\Delta T$ be the inspection interval which is the first decision variable in our model. In the remaining context, we will use $t_{k}\left(t_{k}=k \Delta T\right)$ and $k \Delta T$ interchangeably. Inspection cost $c_{i}$ is incurred at each inspection epoch. Compared with the operation horizon of the system, inspection is assumed to be instantaneous and non-destructive.

Two maintenance actions are considered in this paper: corrective replacement and preventive replacement. At inspection epoch $t_{k}$, if the system functions, the decision maker may decide whether to replace the system preventively or wait till the next inspection. Preventive replacement is carried out when the system is anticipated to approach the failure state, with preventive replacement cost $c_{p}$. Otherwise, the system is left as it be. If the system is found failed at inspection, then it is correctively replaced, with corrective replacement cost $c_{r}$. A replacement can either be a physical replacement or an overhaul that restores the system to the as-good-as-new state. Although both corrective replacement and preventive replacement bring the system to the as-good-as-new state, their cost may differ because corrective replacement is unplanned, which requires more logistic support and disturbs the operation schedule. In addition, failure may incur additional costs such as damage to the environment, which is included in the corrective replacement cost. It is therefore anticipated $c_{r}>c_{p}$. Since the system is operating with unsatisfied performance during the interval from system failure to the next inspection, a downtime cost is charged per unit time, denoted as $c_{d}$. The sensor is replaced along with system replacement.

If the inspection is perfect, then the optimal maintenance policy turns out as a control limit policy, which states that the system is preventively replaced when the observed system state exceeds the optimal preventive replacement threshold. However, the control limit policy based on perfect inspection may not remain optimal in presence of measurement errors. 
This is due to the fact the true system state cannot be fully captured at inspection, rather, what can be obtained is a normally distributed random estimate, whose behavior depends on two parameters: mean and variance. A maintenance decision solely based on the mean of system state may lead to a suboptimal solution. It is well noted that under perfect inspection, the optimal replacement policy is to replace the system when its degradation level hits a constant threshold. However, under imperfect inspection, as the underlying degradation is estimated rather than directly observed, maintenance decision has to take into account the effect of mean and variance of the estimated system state. Fig. 2 describes the difference of preventive replacement under perfect inspection and imperfect inspection. Under imperfect inspection, a more conservative maintenance policy is warranted to balance the influence of estimation uncertainty.

Let $R_{t_{k}}\left(t \mid \mathbf{Y}_{1: k}\right)$ be the system reliability function given the observation history $\mathbf{Y}_{1: k}$. In the case of perfect inspection, due to the memoryless property of Wiener process, $R_{t_{k}}\left(t \mid \mathbf{Y}_{1: k}\right)$ is identical to the reliability function given the current system state, $R_{t_{k}}\left(t \mid \mathbf{Y}_{1: k}\right)=R_{t_{k}}\left(t \mid x_{k}\right)$. However, in the present case where inspection contains noise and reliability estimation has to rely on the whole observation history, the Markov property no longer holds.

The maintenance policy works as follows: at the $k$ th inspection, if the system has failed, corrective replacement is implemented. If the system is still functioning, a preventive replacement is carried out if the system reliability at the next inspection epoch $R_{t_{k}}\left(t_{k}+\Delta T \mid \mathbf{Y}_{1: k}\right)$ falls below a critical threshold $R_{s}\left(R_{t_{k}}\left(t_{k}+\Delta T \mid \mathbf{Y}_{1: k}\right)<R_{s}<1\right)$. Otherwise, it is left unattained. The reliability threshold $R_{s}$ is the second decision variable of maintenance optimization. For safetycritical systems where a high reliability level is warranted, the reliability threshold is given as a constraint in optimization.

\section{B. Maintenance cost model}

Following the tradition of existing maintenance policies, in this paper long run cost rate is employed as the criterion to evaluate the effectiveness of the proposed maintenance policy. The long run cost rate is given as

$$
C^{\infty}\left(\Delta T, R_{s}\right)=\lim _{t \rightarrow \infty} \frac{C(t)}{t}
$$

Based on the proposed maintenance policy, it follows

$$
C(t)=c_{i} N_{I}(t)+c_{p} N_{P}(t)+c_{c} N_{C}(t)+c_{d} W(t)
$$

where $N_{I}(t), N_{P}(t)$, and $N_{C}(t)$ are respectively the number of inspection, preventive replacement and corrective replacement in the time interval $[0, t], W(t)$ is the cumulative downtime. The objective of the maintenance optimization is to minimize the long run cost rate by searching the optimal inspection interval $\Delta T$ and reliability threshold $R_{s}$.

Since both preventive replacement and corrective replacement restore the system to the as-good-as-new state, the degradation process $\{X(t) ; t \geq 0\}$ is a regenerative process. A renewal cycle occurs when the system is replaced. A renewal cycle is defined as the time interval between two consecutive replacements or the time period to the first replacement since system installation. The classical renewal-reward theorem can be applied to calculate the long run maintenance cost rate of (7) [26], [42], which is given as

$$
\begin{aligned}
& C^{\infty}\left(\Delta T, R_{S}\right)=\frac{E[C(\mathrm{Z})]}{E[\mathrm{Z}]} \\
& =\frac{c_{i} E\left[N_{I}(\mathrm{Z})\right]+c_{p} E\left[N_{P}(\mathrm{Z})\right]+c_{c} E\left[N_{C}(\mathrm{Z})\right]+c_{d} E[W(\mathrm{Z})]}{E[\mathrm{Z}]}
\end{aligned}
$$

where $\mathrm{Z}$ is the length of a renewal cycle, and $C(\mathrm{Z})$ is the total maintenance cost of a renewal cycle, $N_{I}(\mathrm{Z}), N_{P}(\mathrm{Z})$, and $N_{C}(\mathrm{Z})$ are respectively the number of inspection, preventive replacement and corrective replacement in a renewal cycle, $W(\mathrm{Z})$ is the cumulative downtime of a renewal cycle.

Let $p_{c}(k)$ be the probability that the renewal cycle ends with corrective replacement at the $k$ th inspection, and $p_{p}(k)$ the probability that the renewal cycle ends with preventive replacement at the $k$ th inspection. By simple algebra, we can rewrite the long run cost rate as

$$
\begin{aligned}
& C^{\infty}\left(\Delta T, R_{s}\right)=\frac{c_{i}}{\Delta T}+c_{d} \sum_{k=1}^{\infty} p_{c}(k) \\
& +\frac{c_{p} \sum_{k=1}^{\infty} p_{p}(k)+c_{c} \sum_{k=1}^{\infty} p_{c}(k)-c_{d} \sum_{k=1}^{\infty} p_{c}(k) \int_{(k-1) \Delta T}^{k \Delta T} t d F_{T}(t)}{\Delta T \sum_{k=1}^{\infty} k\left(p_{c}(k)+p_{p}(k)\right)}
\end{aligned}
$$

Denote $A_{k}$ as the event that corrective replacement is carried out at the $k$ th inspection and $B_{k}$ the event that preventive replacement is performed at the $k$ th inspection. $p_{c}(k)$ and $p_{p}(k)$ can be expressed as

$$
p_{c}(k)=P\left(A_{k} \bigcap_{i=1}^{k-1} \bar{A}_{i} \bar{B}_{i}\right)
$$

and

$$
p_{p}(k)=P\left(B_{k} \bigcap_{i=1}^{k-1} \bar{A}_{i} \bar{B}_{i}\right)
$$

Since preventive replacement is performed when the oneinspection-ahead reliability at inspection exceeds the threshold $R_{s}$, one first has to calculate $R_{t_{k}}\left(t_{k}+\Delta T\right)$ at $k$ th inspection before reaching $p_{c}(k)$ and $p_{p}(k)$, which is denoted as

$$
\begin{aligned}
R_{t_{k}}\left(t_{k}+\Delta T\right) & =E_{\mathbf{Y}_{1: k}}\left[R_{t_{k}}\left(t_{k}+\Delta T \mid \mathbf{Y}_{1: k}\right)\right] \\
& =\int_{y_{1}} \int_{y_{2}} \ldots \int_{y_{k}} R_{t_{k}}\left(t_{k}+\Delta T \mid y_{1}, y_{2}, \ldots y_{k}\right) . \\
& f\left(y_{1}, y_{2}, \ldots y_{k}\right) d y_{1} d y_{2} \ldots d y_{k}
\end{aligned}
$$

where $f\left(y_{1}, y_{2}, \ldots y_{k}\right)$ is the joint distribution of $y_{1}, y_{2}, \ldots y_{k}$. For the system subject to imperfect inspection, Kalman filter is used to progressively estimate the system state and reliability. In the following, we will present the procedure for state estimation and reliability prediction given the observation history. However, since state estimation via Kalman filter depends on the measurement history, computation of the one-inspectionahead reliability has to integrate all possible measurements $\mathbf{Y}_{1: k}$. It is extremely difficult to obtain the analytical expression of $R_{t_{k}}\left(t_{k}+\Delta T\right)$, let alone the long run cost rate of (9). 


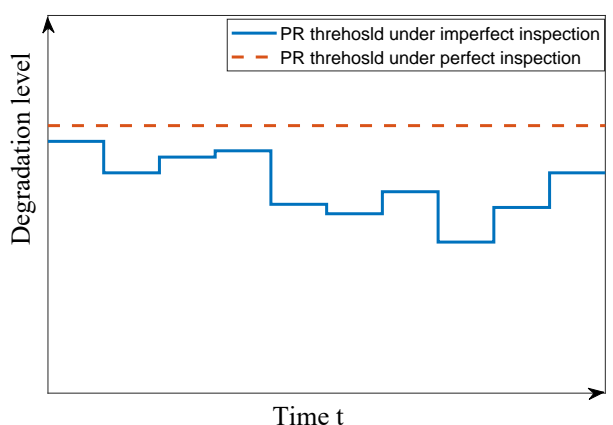

Fig. 2: Comparison of preventive replacement under perfect and imperfect inspection

Therefore, we resort to Monte Carlo simulation to evaluate the maintenance policy. An optimal policy is achieved by minimizing the long run cost rate in (9), i.e.,

$$
\left\{\Delta T^{*}, R_{s}^{*}\right\}=\underset{\left(\Delta T, R_{s}\right)}{\arg \min }\left\{C^{\infty}\left(\Delta T, R_{s}\right) ; 0<R_{s}<1\right\}
$$

Note that for some systems the inspection interval is given as a constraint due to industrial standards. With respect to the case where the inspection interval is given in advance, the decision variable is reduced to the reliability threshold. The optimal maintenance decision $\left\{\Delta T^{*}, R_{s}^{*}\right\}$ can be obtained via two-directional search. A near-optimal initial guess of $\left\{\Delta T^{*}, R_{s}^{*}\right\}$ contributes to facilitating the search algorithm. The near-optimal initial guess of the optimal maintenance decision is presented in Appendix A.

Remark 3.1: In the current work, periodic inspection is used to observe the system state. In some applications, the system may be subject to continuous monitoring, where a dedicated sensor is installed along with the system to monitor its health condition [24], [25]. In this scenario, the observations are presented at every basic time unit, which implies $\Delta T=\omega$ for a small value $\omega$ (e.g., $\omega=0.01)$. In addition, the inspection cost will be suppressed, $c_{i}=0$. But we need to incorporate the one-time sensor installation cost during the formulation of maintenance cost. It should be noticed that this is a special case of our proposed maintenance policy which can be obtained by setting the inspection cost $c_{i}=0$ and $\Delta T=\omega$. In the case of continuous monitoring, the maintenance cost of (8) is reduced to

$$
C(t)=c_{p} N_{P}(t)+c_{c} N_{C}(t)+c_{0}
$$

where $c_{0}$ is the one-time installation cost of the sensor. For continuous monitoring, it should be noted that the one-stepahead reliability would approach to $1, R_{S} \rightarrow 1$, since the inspection interval $\Delta T \rightarrow 0$. On the other hand, for continuous monitoring, the maintenance lead time (i.e., the time interval between the maintenance alarm triggered and the actual maintenance time) should be taken into account. Therefore, the reliability criterion is modified based on the lead time. Let $R_{c}$ be the system reliability evaluated ahead of the lead time $T_{L}$. The long run cost rate is then given as

$$
C^{\infty}\left(R_{c}\right)=\frac{c_{p} E\left[N_{P}(\mathrm{Z})\right]+c_{c} E\left[N_{C}(\mathrm{Z})\right]+c_{0}}{E[\mathrm{Z}]}
$$

\section{ONLINE STATE ESTIMATION}

Now that we have calculated the optimal maintenance decision variables, we are now arriving at implementing the maintenance actions based on the observation history. As a first step we need to estimate the degradation state of the system and sensor. Kalman filter serves for the estimation purpose. Let $s_{k}=S\left(t_{k}\right)$ represent the sensor state at time $t_{k}$. The set of system degradation and sensor degradation are expressed as $\mathbf{X}_{1: k}=\left\{x_{1}, x_{2}, \ldots, x_{k}\right\}$ and $\mathbf{S}_{1: k}=\left\{s_{1}, s_{2}, \ldots, s_{k}\right\}$. With the aforementioned notations, we can have the state-space model as

$$
\left\{\begin{array}{l}
x_{k}=x_{k-1}+\lambda\left(t_{k}-t_{k-1}\right)+u_{k} \\
s_{k}=s_{k-1}+\eta\left(t_{k}-t_{k-1}\right)+v_{k} \\
y_{k}=x_{k}+s_{k}+\varepsilon_{k}
\end{array}\right.
$$

where $u_{k}=\sigma[B(k)-B(k-1)]$ and $v_{k}=\delta[B(k)-B(k-1)]$. $\left\{u_{k}, k \geq 0\right\},\left\{v_{k}, k \geq 0\right\}$ and $\left\{\varepsilon_{k}, k \geq 0\right\}$ follow statistically independent and identically normal distribution, i.e., $u_{k} \sim N\left(0, \sigma^{2}\left(t_{k}-t_{k-1}\right)\right), v_{k} \sim N\left(0, \delta^{2}\left(t_{k}-t_{k-1}\right)\right)$ and $\varepsilon_{k} \sim$ $N\left(0, \gamma^{2}\right)$.

The underlying system degradation state is casted by the sensor degradation variability and measurement uncertainty and can only be estimated based on the observations up to time $t, \mathbf{Y}_{1: k}$. Since (4) exhibits dynamic linear property and the degradation variability, $u_{k}$ and $v_{k}$, and measurement noise $\varepsilon_{k}$, follow Gaussian distribution, Kalman filter can be employed to estimate the system and sensor degradation states. Kalman filter is known as linear quadratic estimation and has shown its effectiveness in various applications [12], [43]-[45]. Under the framework of Kalman filter, we reorganize the state-space model as

$$
\left\{\begin{aligned}
\mathbf{z}_{k} & =\mathbf{A} \mathbf{z}_{k-1}+\mathbf{B}_{k}+\mathbf{w}_{k} \\
y_{k} & =\mathbf{H z} \mathbf{z}_{k}+\varepsilon_{k}
\end{aligned}\right.
$$

where $\mathbf{z}_{k}=\left[\begin{array}{c}x_{k} \\ s_{k}\end{array}\right], \mathbf{A}=\left[\begin{array}{ll}1 & 0 \\ 0 & 1\end{array}\right], \mathbf{B}_{k}=\left[\begin{array}{c}\lambda\left(t_{k}-t_{k-1}\right) \\ \eta\left(t_{k}-t_{k-1}\right)\end{array}\right]$, $\mathbf{H}=\left[\begin{array}{ll}1 & 1\end{array}\right], \quad \mathbf{w}_{k} \in R^{2 \times 1}, \quad \mathbf{w}_{k} \sim N\left(0, \mathbf{Q}_{k}\right), \quad \mathbf{Q}_{k}=$ $\left[\begin{array}{cc}\sigma^{2}\left(t_{k}-t_{k-1}\right) & 0 \\ 0 & \delta^{2}\left(t_{k}-t_{k-1}\right)\end{array}\right]$.

As the first step, we define the expectation and variance of the estimators $\mathbf{Z}_{\mathbf{k}}$ conditional on the observation history $\mathbf{Y}_{1: k}$, which is given as

$$
\begin{gathered}
\hat{\mathbf{z}}_{k \mid k}=\left[\begin{array}{c}
\hat{x}_{k \mid k} \\
\hat{s}_{k \mid k}
\end{array}\right]=E\left(\mathbf{z}_{k} \mid \mathbf{Y}_{1: k}\right) \\
\mathbf{P}_{k \mid k}=\left[\begin{array}{cc}
\chi_{x, k}^{2} & \chi_{x s, k}^{2} \\
\chi_{x s, k}^{2} & \chi_{s, k}^{2}
\end{array}\right]=\operatorname{cov}\left(\mathbf{z}_{k} \mid \mathbf{Y}_{1: k}\right)
\end{gathered}
$$

where $\hat{x}_{k \mid k}=E\left(x_{k} \mid \mathbf{Y}_{1: k}\right), \hat{s}_{k \mid k}=E\left(s_{k} \mid \mathbf{Y}_{1: k}\right), \chi_{x, k}^{2}=\operatorname{var}\left(x_{k} \mid \mathbf{Y}_{1: k}\right)$, $\chi_{s, k}^{2}=\operatorname{var}\left(s_{k} \mid \mathbf{Y}_{1: k}\right)$, and $\chi_{x s, k}^{2}=\operatorname{cov}\left(x_{k} s_{k} \mid \mathbf{Y}_{1: k}\right)$. In addition, the one-step-ahead predicted estimation and variance of $\mathbf{z}_{\mathbf{k}}$ are denoted as

$$
\hat{\mathbf{z}}_{k \mid k-1}=\left[\begin{array}{c}
\hat{x}_{k \mid k-1} \\
\hat{s}_{k \mid k-1}
\end{array}\right]=E\left(\mathbf{z}_{k} \mid \mathbf{Y}_{1: k-1}\right)
$$




$$
\mathbf{P}_{k \mid k-1}=\left[\begin{array}{cc}
\chi_{x, k \mid k-1}^{2} & \chi_{x s, k \mid k-1}^{2} \\
\chi_{x s, k \mid k-1}^{2} & \chi_{s, k \mid k-1}^{2}
\end{array}\right]
$$

At the $k$ th inspection time $t_{k}$, we can have the following Kalman filter procedure:

1) State estimation

State prediction:

$$
\hat{\mathbf{z}}_{k \mid k-1}=\mathbf{A} \hat{\mathbf{z}}_{k-1 \mid k-1}+\mathbf{B}_{k}
$$

Updated state estimate:

$$
\hat{\mathbf{z}}_{k \mid k}=\hat{\mathbf{z}}_{k \mid k-1}+\mathbf{K}(k)\left(y_{k}-\mathbf{H} \hat{\mathbf{z}}_{k \mid k-1}\right)
$$

2) State covariance estimation

Covariance prediction:

$$
\mathbf{P}_{k \mid k-1}=\mathbf{A} \mathbf{P}_{k-1 \mid k-1} \mathbf{A}^{T}+\mathbf{Q}_{k}
$$

Filter gain:

$$
\mathbf{K}(k)=\mathbf{P}_{k \mid k-1} \mathbf{H}^{T}\left[\mathbf{H P}_{k \mid k-1} \mathbf{H}^{T}+\gamma^{2}\right]^{-1}
$$

Updated state covariance:

$$
\mathbf{P}_{k \mid k}=\mathbf{P}_{k \mid k-1}-\mathbf{K}(k) \mathbf{H P}_{k \mid k-1}
$$

The initial values of the degradation states are given as

$$
\hat{\mathbf{z}}_{0 \mid 0}=\left[\begin{array}{l}
0 \\
0
\end{array}\right], \mathbf{P}_{k \mid k}=\left[\begin{array}{ll}
0 & 0 \\
0 & 0
\end{array}\right]
$$

Since the degradation variability and measurement noise are normally distributed, it can be concluded the posterior estimation of the system and sensor degradation state conditional on measurement history $\mathbf{Y}_{1: k}$ follows a bivariate Gaussian distribution, $\mathbf{z}_{k} \mid \mathbf{Y}_{1: k} \sim N\left(\hat{\mathbf{z}}_{k \mid k}, \mathbf{P}_{k \mid k}\right)$. In particular, we have

$$
\begin{aligned}
& x_{k} \mid \mathbf{Y}_{1: k} \sim N\left(\hat{x}_{k \mid k}, \chi_{x, k}^{2}\right) \\
& s_{k} \mid \mathbf{Y}_{1: k} \sim N\left(\hat{s}_{k \mid k}, \chi_{s, k}^{2}\right)
\end{aligned}
$$

Without assuming a deterministic sensor drift parameter $\eta$, in the case where $\eta$ is random in nature, state estimation procedure has to incorporate the random effect of $\eta$. If $\eta$ is normally distributed, $\eta \sim N\left(\mu_{\eta}, \sigma_{\eta}\right)$, Kalman filter can be applied as well. The detailed Kalman filter procedure is shown in Appendix B.

Remark 4.1: For some systems, the system degradation may exert influence on the sensor degradation process due to the specific inspection mechanism and environment. However, the underlying physical mechanism may be very complicated to prohibit an accurate modeling. For illustrative purpose, we present the procedure of state estimation under the case where the system degradation has an additive impact on sensor degradation. Details are presented in Appendix C.

Following the concept of FPT, the remaining useful time (RUL) of the system at the $k$ th inspection time $t_{k}, L_{k}$, is defined as

$$
L_{k}=\inf \left\{l_{k}: X\left(l_{k}+t_{k}\right) \geq \zeta\right\}
$$

By use of Kalman filter, given the measurement history $\mathbf{Y}_{1: k}$, the distribution of RUL can be obtained as

$$
\begin{aligned}
& f_{L_{k} \mid \mathbf{Y}_{1: k}}(t)=E_{x_{k}}\left[f_{L_{k}}\left(t \mid x_{k}\right)\right] \\
& =\frac{\left(\zeta-\hat{x}_{k \mid k}\right) \sigma^{2}+\chi_{x, k}^{2} \lambda}{\sqrt{2 \pi\left(\chi_{x, k}^{2}+\sigma^{2} t\right)^{3}}} \exp \left(-\frac{\left(\zeta-\hat{x}_{k \mid k}-\lambda t\right)^{2}}{2\left(\chi_{x, k}^{2}+\sigma^{2} t\right)}\right)
\end{aligned}
$$

and

$$
\begin{aligned}
F_{L_{k} \mid \mathbf{Y}_{1: k}}(t) & =\int F_{L_{k}}\left(t \mid x_{k}\right) f_{\phi}\left(x_{k}\right) d x_{k} \\
& =1-\Phi\left(\frac{\zeta-\hat{x}_{k \mid k}-\lambda t}{\sqrt{\chi_{x, k}^{2}+\sigma^{2} t}}\right) \\
& +\exp \left(\frac{2 \lambda\left(\zeta-\hat{x}_{k \mid k}\right)}{\sigma^{2}}+\left(\frac{\sqrt{2} \lambda \chi_{x, k}}{\sigma^{2}}\right)^{2}\right) . \\
& \Phi\left(\frac{-\zeta+\hat{x}_{k \mid k}-\lambda t-2 \lambda \frac{\chi_{x, k}^{2}}{\sigma^{2}}}{\sqrt{\chi_{x, k}^{2}+\sigma^{2} t}}\right)
\end{aligned}
$$

where $f_{L_{k} \mid \mathbf{Y}_{1: k}}(t)$ and $F_{L_{k} \mid \mathbf{Y}_{1: \mathbf{k}}}(t)$ are the conditional pdf and cdf of the RUL of the system, $f_{\phi}\left(x_{k}\right)$ is the pdf of system state at the $k$ th inspection,

$$
f_{\phi}\left(x_{k}\right)=\frac{1}{\sqrt{2 \pi \chi_{x, k}^{2}}} \exp \left(\frac{\left(x-\hat{x}_{k \mid k}\right)^{2}}{2 \chi_{x, k}^{2}}\right)
$$

The dynamic maintenance is implemented by comparing the estimated one-inspection-ahead system reliability with the calculated threshold $R_{s}^{*}$. However, if the sensor fails, the maintenance decision has to be made based the previous observations. Details of the impact of sensor failure on maintenance decision making is provided in Appendix D.

In the previous discussion, we assume that the parameters of both the system and sensor degradation processes are known in advance. In practice, the degradation parameters can be estimated from historical degradation data. To estimate the Wiener process parameters based on real data, which has to include both $S(t)$ and $X(t)$, we need to have two sensors, one with degradation and the other without any degradation. The one without degradation is used to estimate the parameters of the sensor degradation. Based on the estimated sensor degradation parameters, the signals from the degraded sensor are then used to estimate the parameters of system degradation. One single sensor fails to simultaneously estimate the system and sensor degradation parameters, as the degradation information of the system and sensor is mixed. With two sensors we can estimate the parameters. In Appendix E, we present the procedure to estimate the sensor degradation parameters in presence of a new sensor (without any degradation).

\section{APPLICATION IN WASTEWATER TREATMENT PLANTS}

In this section, a wastewater treatment plant is used to illustrate the proposed maintenance policy and parameter estimation. Activated sludge process is a widely adopted to handle pollutants in wastewater treatment plants. However, scheduled operation of activated sludge process is often impeded in presence of filamentous bulking. Sludge bulking occurs largely due to the growth of filamentous bacteria, which can be modeled as a degradation process [46], [47]. In practice, an empirical measurement, Sludge Volume Index (SVI), is commonly used to characterize the degradation of filamentous sludge bulking. Unfortunately the real data is not available. The example we use is a real problem that serves the purpose of illustration. 


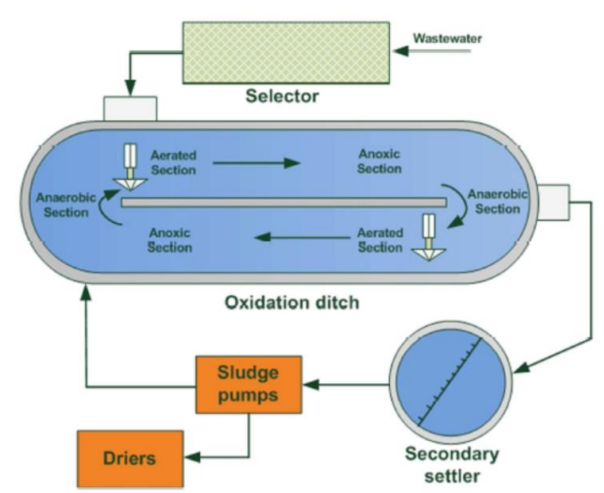

Fig. 3: Sketch of oxidation ditch process

\section{A. Case study setting}

It is assumed that the SVI follows a Wiener process with linear drift, where $\lambda=1$ and $\sigma=0.5$. The active sludge process is considered failed when the SVI exceeds a prespecified level. Note that determination of an accurate failure threshold to indicate serious filamentous sludge bulking is still an open issue. For illustrative purpose, an arbitrary value of SVI is used as failure threshold in this paper, $\zeta=15$. Among various active sludge processes, oxidation ditch process is a biological treatment process that utilizes long solids retention time to achieve satisfactory nitrogen removal performance. Fig. 3 shows a schematic of the oxidation ditch process [47]. On the other hand, due to the existence of filamentous bacteria and corrosive materials in the wastewater, the sensor dedicated to inspecting the degradation level of sludge bulking is subject to degradation. The sensor itself is assumed to suffer a Wiener degradation process with $\eta=0.2$ and $\delta=0.1$. Observation at inspection is not only influenced by the system degradation and sensor degradation, but also contaminated by noise with $\gamma=0.5$.

The wastewater is periodically inspected to determine the degradation level (SVI), with inspection cost $c_{i}=1$. When the SVI hits the preventive replacement threshold, the wastewater is intervened preventively with cost $c_{p}=20$. If the SVI is found to exceed the failure threshold, which implies a serious filamentous sludge bulking, the wastewater is treated with large effort, at the cost $c_{r}=50$. In addition, during the period from system failure to the next inspection, the oxidation ditch process is operating under serious sludge bulking, which incurs cost $c_{d}=200$ per unit time.

\section{B. Key results}

1) Numerical results: In the presence of sensor degradation and imperfect observation, the optimal reliability threshold cannot be analytically calculated. Monte Carlo simulation is therefore employed. The number of Monte Carlo simulation is 5000. The optimal maintenance policy is achieved at $\Delta T^{*}=3$ and $R_{s}^{*}=0.999$, with the long run cost rate $C^{\infty *}=2.72$. Fig. 4 and Fig. 5 show how the one-inspection-ahead reliability $R_{S}$ varies with respect to the estimated system state $\hat{x}$ and the associated variance $\chi^{2}$. It is clearly observed that $R_{s}$ shows a decreasing trend with $\hat{x}$ and $\chi^{2}$.

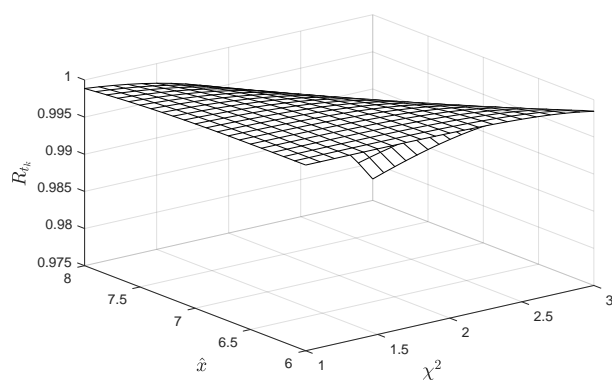

Fig. 4: One-inspection-ahead system reliability
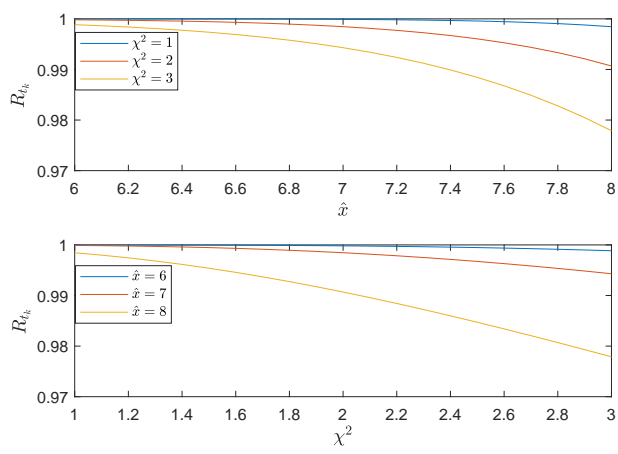

Fig. 5: Variation of $R_{t_{k}}$ with $\hat{x}$ and $\chi^{2}$ respectively

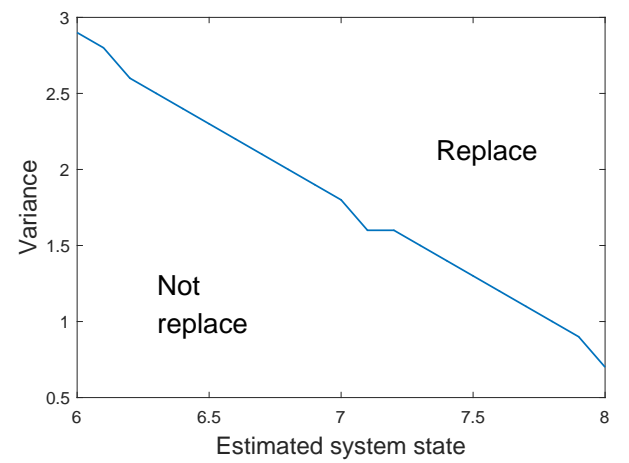

Fig. 6: Maintenance action in terms of $\hat{x}$ and $\chi^{2}$

As can be observed though the numerical example, the long run cost rate under perfect inspection is $2.62\left(C^{(0) \infty}=2.62\right)$, which is close to the optimal maintenance cost under sensor degradation $\left(C^{\infty *}=2.72\right)$. This indicates that with the proposed state estimation method and the condition-based maintenance, the negative impact of sensor degradation can be effectively addressed.

Since preventive maintenance action depends on the predicted system reliability, which, however, is determined by the mean and variance of the estimated system state. To facilitate maintenance decisions at inspection, Fig. 6 depicts the boundary for preventive replacement in terms of the mean and variance of the estimated system state.

According to the reliability threshold for preventive replacement, the maintenance action at each inspection can be obtained by comparing the estimated one-inspection-ahead system reliability with the threshold. Table I presents the maintenance actions and associated quantities at each inspection, where the inspection interval is $\Delta T=3$. Note that the measure- 
TABLE I: Illustration of maintenance decisions at inspection

\begin{tabular}{cccccc}
\hline$k$ & $y_{k}$ & $\hat{x}_{k \mid k}$ & $\chi_{k \mid k}^{2}$ & $R_{t_{k}}$ & Decision \\
\hline 1 & 4 & 3 & 0.2039 & 1 & Do nothing \\
2 & 6 & 6 & 0.2414 & 1 & Do nothing \\
3 & 13 & 9 & 0.2706 & 0.9981 & Preventive replacement \\
\hline
\end{tabular}

ment history $\mathbf{Y}_{k}$ is randomly generated for illustration purpose. As can be observed, at the third inspection, the estimated system reliability drops below the threshold $R_{s}^{*}=0.999$, and preventive replacement is carried out to prevent the system from failure.

2) Key findings: To illustrate the impact of sensor degradation, we compare the proposed maintenance policy with the one that disregards the sensor degradation in spite of its existence. For the maintenance policy that simply compares the measurement value with the preventive replacement threshold under perfect inspection, the long run cost rate turns out as $C^{(1) \infty}=4.27$. This is due to the fact that preventive replacement solely based on the observation leads to a far more conservative policy and unnecessary intervention increases the maintenance cost. By comparison, the long run cost rate of the maintenance policy considering the sensor degradation is $C^{\infty}=2.72$, which indicates that the sensor degradation has a significant impact on the optimal maintenance policy and should be taken into account for maintenance decision-making.

Additionally, we plot in Fig. 7 the variation of maintenance cost with respect to the sensor degradation rate $\eta$. It can be found that our policy provides a stable maintenance cost (around 2.7) in spite of the variation of sensor degradation rate, while the maintenance cost under the policy (Policy I) that disregards the sensor degradation exhibits an increasing trend with $\eta$. Comparison from Fig. 7 implies that our approach is more effective in cases where the sensor exhibits a serious degradation process. Obviously if the sensor degradation is negligible compared with the system degradation, then our model will not be encouraged. We would suggest adopting our model for maintenance decision-making if the systems and the dedicated sensors are operating under extreme conditions (e.g., high temperature, high humidity, corrosive surroundings, etc.) where the sensor degradation exerts a significant impact. However, it should be noted a system may fail due to various mechanisms, which may not exhibit the degradation pattern. For example, external shocks may lead to sudden failure of an operating system. In this case, we have to resort to other models, since our model is applicable to model the gradual degradation process, while fails to capture the influence of external shock.

In addition, we compare the proposed approach with the existing methods to show the impact of sensor degradation on maintenance cost. In particular, we compare with a filtering approach which is adopted to address time-varying noise variance [48]. The maintenance cost with the approach in [48] is $C^{(2) \infty}=4.35$, which is close to the maintenance policy that simply compares measurements with the threshold under perfect inspection $\left(C^{(1) \infty}=4.27\right)$. Admittedly, the approach can effectively deal with the measurement noise, which, however, fails to distinguish the sensor degradation and

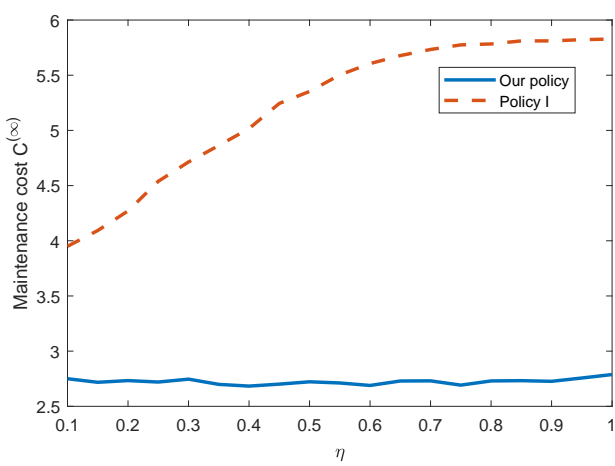

Fig. 7: Variation of $C^{\infty}$ on sensor degradation rate

TABLE II: Initial guess of optimal $T^{(0)}$ and $M$

\begin{tabular}{|c|c|c|c|c|c|c|c|}
\hline & & \multicolumn{6}{|c|}{$T^{(0)}$} \\
\hline & & 3.2 & 3.4 & 3.6 & 3.8 & 4 & 4.2 \\
\hline \multirow{10}{*}{$M$} & 2.5 & 5.94 & 5.79 & 2.95 & 5.29 & 5.08 & 4.88 \\
\hline & 3 & 5.31 & 5.21 & 2.85 & 5.01 & 4.90 & 4.71 \\
\hline & 3.5 & 4.53 & 4.56 & 2.72 & 4.57 & 4.60 & 4.45 \\
\hline & 4 & 3.93 & 3.99 & 2.67 & 4.14 & 4.18 & 4.16 \\
\hline & 4.5 & 3.56 & 3.70 & $2.62 *$ & 3.91 & 4.08 & 4.14 \\
\hline & 5 & 3.62 & 3.59 & 3.08 & 3.90 & 4.24 & 4.25 \\
\hline & 5.5 & 3.72 & 3.71 & 3.48 & 4.46 & 4.44 & 4.97 \\
\hline & 6 & 3.89 & 3.98 & 3.97 & 5.12 & 5.57 & 5.74 \\
\hline & 6.5 & 4.57 & 4.56 & 5.34 & 6.16 & 6.87 & 7.13 \\
\hline & 7 & 5.53 & 5.52 & 6.03 & 7.05 & 8.36 & 9.23 \\
\hline
\end{tabular}

system degradation in presence of sensor drift. The existing filtering methods are applicable to 0-mean measurement noise. However, in our case, the measurement is a mixture of system degradation, sensor degradation, and the measurement noise. Existing methods can handle the unbiased measurement noise. However, in presence of the sensor drift, the measurement is biased. Therefore, we have to estimate sensor drift as a first step so as to provide an accurate estimation of the system sate.

\section{Discussion}

1) Initial guess of the inspection interval and preventive replacement threshold: To achieve the optimal maintenance decision under imperfect inspection, the initial guess of the inspection interval and one-inspection-ahead reliability is obtained as a first step. Table II presents how the long-run cost rate under perfect inspection $C^{(0) \infty}$ varies with different inspection interval $T^{(0)}$ and preventive replacement threshold $M$. It can be observed that without sensor degradation and measurement noise, the optimal maintenance policy is achieved at $\Delta T^{(0)}=3.6$ and $M=4.5$, with the long run cost rate $C^{(0) \infty}=2.62$. The one-inspection-ahead reliability under this scenario $R_{s}^{(0)}$ is close to 1 .

2) Sensitivity analyses: Compared with perfect inspection, the influence of imperfect inspection lies in the uncertainty of the degradation and measurement process. Therefore, it is interesting to investigate how the optimal long run cost rate varies with the variance parameters. Fig. 8 shows the variation of $C^{\infty}$ with respect to the variance parameters: $\sigma, \delta$ and $\gamma$. Since a larger $\sigma$ leads to more uncertainty of the degradation process, the long run cost rate $C^{\infty}$ increases with the diffusion parameter $\sigma$. In addition, $C^{\infty}$ is largely affected by $\sigma$, while $\delta$ 

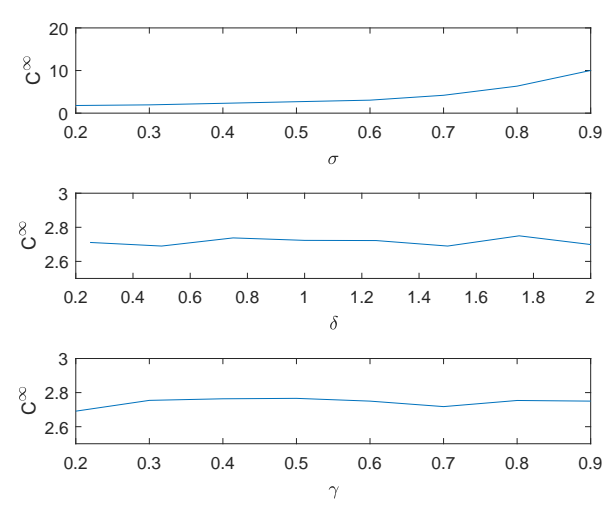

Fig. 8: Variation of $C^{\infty}$ on variance parameters
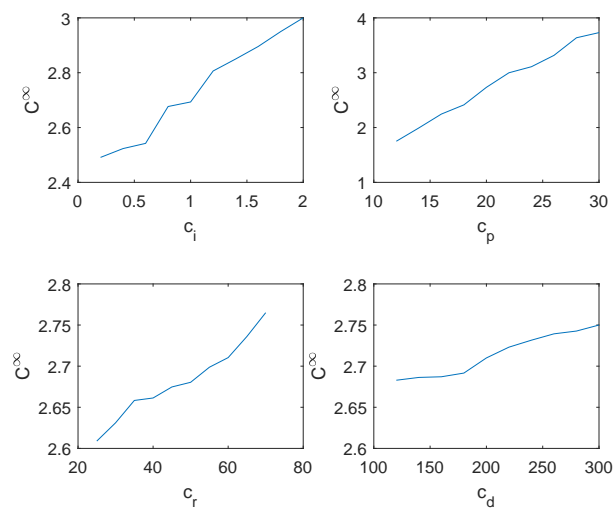

Fig. 9: Variation of $C^{\infty}$ on cost parameters

and $\gamma$ have little impact on $C^{\infty}$. This is due to the fact that $\sigma$ determines the variation of the degradation process. A large $\sigma$ significantly increases the randomness of system failure time and system state at inspection. On the other hand, the effect of $\delta$ and $\gamma$ is diminished by Kalman filter, which, to some extent, indicates the effectiveness of Kalman filter.

In addition, we are interested to investigate the effect of cost parameters on the long run cost rate $C^{\infty}$. Fig. 9 shows how $C^{\infty}$ varies with the cost parameters: $c_{i}, c_{p}, c_{r}$ and $c_{d}$. Clearly $C^{\infty}$ increases with the cost parameters. In addition, $c_{i}$ and $c_{p}$ exert more impact on the long run cost rate than $c_{r}$ and $c_{d}$. The underlying logic of the influence can be observed from the reliability threshold for preventive replacement. The system is preventively replaced at high reliability $R_{s}^{*}=0.999$, which leads to a tiny probability of system failure. In other words, under most circumstances, a renewal cycle ends with preventive replacement rather than corrective replacement.

3) Extension to continuous monitoring: The proposed approach can also be extended to continuous monitoring. It should be noted that the one-inspection-head reliability threshold is not applicable for continuous monitoring since the inspection interval is close to 0 . On the other hand, the maintenance lead time will influence the maintenance efficiency and should be taken into account in presence of continuous monitoring. For illustration purpose, we let the lead time $T_{L}=2$. In addition, the inspection cost is set as $c_{i}=0$, and the inspection interval is given as $\Delta T=0.1$. With our method, the minimal maintenance cost $C^{\infty}=2.45$ is achieved at the reliability threshold $R_{C}=0.9998$, which is evaluated ahead of the lead time $T_{L}$. We also compare with the maintenance policy that neglects the sensor degradation in spite of its existence, where the associated maintenance cost is 2.68. Comparison between our approach and the policy that disregards the sensor degradation leads to the conclusion that sensor degradation exerts an influential effect on maintenance policy under continuous monitoring.

\section{CONCLUSION}

This paper develops a condition-based maintenance policy for systems with degraded sensors. Inspection of system state is influenced not only by the system and sensor degradation process, but also the measurement noise. Kalman filter is used to deal with the degradation and measurement uncertainty. Degradation level of the system and sensor is updated at the arrival of a new measurement. A maintenance cost model is constructed as a first step and the optimal maintenance policy is achieved by minimizing the long run cost rate. Under the proposed maintenance policy, optimal inspection interval and reliability threshold are obtained to implement maintenance actions. At each inspection, the maintenance actions are carried out by comparing the estimated system reliability with the corresponding threshold. Application in wastewater treatment plants illustrates the effectiveness of the proposed policy.

It is revealed through the numerical example that if we ignore the sensor degradation while it exists, the maintenance would severely deviate from the optimal one. On the other hand, if we realize the existence of sensor degradation and use appropriate methods to estimate the system state, its negative impact can be effectively diminished.

There are several interesting issues embedded with the maintenance policy subject to sensor degradation that warrant future research. First, in this paper Wiener process is employed to characterize the degradation process of the system and the sensor. For systems that exhibit monotonic degradation processes, alternative degradation models such as Gamma process and inverse Gaussian process can be used instead. Second, the measurement noise is assumed to follow Gaussian distribution and Kalman filter is used thereafter. For the measurement noise that is not normally distributed, we need to seek other filtering approaches such as particle filtering to deal with the nonGaussian noise.

Another perspective should be the investigation on the applicability of the proposed methodology for a real case study with true data. In addition, sensor-related actions (e.g.,sensor repair) can be incorporated into the maintenance policy if the system state cannot be accurately estimated under sensor degradation and the associated maintenance decisions severely deviate from the optimal one. For safety-critical systems (e.g., nuclear power plants), sensor-related actions are warranted since the system reliability has to be estimated in high accuracy.

\section{ACKNOWLEDGMENT}

This work was supported by the ANR LabCOM PHMFACTORY, grant ANR-15-LCV1-0005-01 of the French Re- 
search Agency. We would like to thank all the LabCOM actors through the entities involved: ANR, PREDICT company, MATERALIA cluster, CRAN, CNRS and Lorraine University.

\section{REFERENCES}

[1] B. de Jonge, R. Teunter, and T. Tinga, "The influence of practical factors on the benefits of condition-based maintenance over time-based maintenance," Reliability Engineering \& System Safety, vol. 158, pp. 21-30, 2017.

[2] P. Do, A. Voisin, E. Levrat, and B. Iung, "A proactive conditionbased maintenance strategy with both perfect and imperfect maintenance actions," Reliability Engineering \& System Safety, vol. 133, pp. 22-32, 2015.

[3] S. Wu, F. P. Coolen, and B. Liu, "Optimization of maintenance policy under parameter uncertainty using portfolio theory," IISE Transactions, vol. 49, no. 7, pp. 711-721, 2017.

[4] A. Grall, L. Dieulle, C. Bérenguer, and M. Roussignol, "Continuous-time predictive-maintenance scheduling for a deteriorating system," IEEE Transactions on Reliability, vol. 51, no. 2, pp. 141-150, 2002.

[5] M.-J. Kallen and J. M. van Noortwijk, "Optimal maintenance decisions under imperfect inspection," Reliability Engineering \& System Safety, vol. 90 , no. 2-3, pp. 177-185, 2005.

[6] G. Curcurù, G. Galante, and A. Lombardo, "A predictive maintenance policy with imperfect monitoring," Reliability Engineering \& System Safety, vol. 95, no. 9, pp. 989-997, 2010.

[7] Y.-H. Lin, Y.-F. Li, and E. Zio, "A framework for modeling and optimizing maintenance in systems considering epistemic uncertainty and degradation dependence based on pdmps," IEEE Transactions on Industrial Informatics, vol. 14, no. 1, pp. 210-220, 2018.

[8] R. S. Lim, H. M. La, and W. Sheng, "A robotic crack inspection and mapping system for bridge deck maintenance," IEEE Transactions on Automation Science and Engineering, vol. 11, no. 2, pp. 367-378, 2014

[9] F. Cipollini, L. Oneto, A. Coraddu, A. J. Murphy, and D. Anguita, "Condition-based maintenance of naval propulsion systems: Data analysis with minimal feedback," Reliability Engineering \& System Safety, vol. 177, pp. 12-23, 2018.

[10] N. Chen, Z.-S. Ye, Y. Xiang, and L. Zhang, "Condition-based maintenance using the inverse gaussian degradation model," European Journal of Operational Research, vol. 243, no. 1, pp. 190-199, 2015.

[11] B. Liu, S. Wu, M. Xie, and W. Kuo, "A condition-based maintenance policy for degrading systems with age-and state-dependent operating cost," European Journal of Operational Research, vol. 263, no. 3, pp. 879-887, 2017.

[12] M. Zhang, O. Gaudoin, and M. Xie, "Degradation-based maintenance decision using stochastic filtering for systems under imperfect maintenance," European Journal of Operational Research, vol. 245, no. 2, pp. $531-541,2015$.

[13] J. Poppe, R. N. Boute, and M. R. Lambrecht, "A hybrid conditionbased maintenance policy for continuously monitored components with two degradation thresholds," European Journal of Operational Research, vol. 268, no. 2, pp. 515-532, 2018

[14] Z.-S. Ye, Y. Wang, K.-L. Tsui, and M. Pecht, "Degradation data analysis using wiener processes with measurement errors," IEEE Transactions on Reliability, vol. 62, no. 4, pp. 772-780, 2013.

[15] X. Si, T. Li, Q. Zhang, and X. Hu, "An optimal condition-based replacement method for systems with observed degradation signals," IEEE Transactions on Reliability, 2018.

[16] J. A. Hontelez, H. H. Burger, and D. J. Wijnmalen, "Optimum conditionbased maintenance policies for deteriorating systems with partial information," Reliability Engineering \& System Safety, vol. 51, no. 3, pp. 267-274, 1996.

[17] V. Makis and X. Jiang, "Optimal replacement under partial observations," Mathematics of Operations Research, vol. 28, no. 2, pp. 382-394, 2003.

[18] M. L. Neves, L. P. Santiago, and C. A. Maia, "A condition-based maintenance policy and input parameters estimation for deteriorating systems under periodic inspection," Computers \& Industrial Engineering, vol. 61, no. 3, pp. 503-511, 2011.

[19] X. Liu, J. Li, K. N. Al-Khalifa, A. S. Hamouda, D. W. Coit, and E. A. Elsayed, "Condition-based maintenance for continuously monitored degrading systems with multiple failure modes," IIE Transactions, vol. 45, no. 4, pp. 422-435, 2013.

[20] R. Srinivasan and A. K. Parlikad, "Semi-markov decision process with partial information for maintenance decisions," IEEE Transactions on Reliability, vol. 63, no. 4, pp. 891-898, 2014.
[21] J. A. Flory, J. P. Kharoufeh, and D. T. Abdul-Malak, "Optimal replacement of continuously degrading systems in partially observed environments," Naval Research Logistics (NRL), vol. 62, no. 5, pp. 395 415,2015

[22] M. Zhang and M. Revie, "Continuous-observation partially observable semi-markov decision processes for machine maintenance," IEEE Transactions on Reliability, vol. 66, no. 1, pp. 202-218, 2017.

[23] S. Yang, "A condition-based failure-prediction and processing-scheme for preventive maintenance," IEEE Transactions on Reliability, vol. 52, no. 3, pp. 373-383, 2003.

[24] A. H. Elwany, N. Z. Gebraeel, and L. M. Maillart, "Structured replacement policies for components with complex degradation processes and dedicated sensors," Operations Research, vol. 59, no. 3, pp. 684-695, 2011.

[25] K. A. Kaiser and N. Z. Gebraeel, "Predictive maintenance management using sensor-based degradation models," IEEE Transactions on Systems, Man, and Cybernetics-Part A: Systems and Humans, vol. 39, no. 4, pp. 840-849, 2009.

[26] K. T. Huynh, A. Barros, and C. Bérenguer, "Maintenance decisionmaking for systems operating under indirect condition monitoring: value of online information and impact of measurement uncertainty," IEEE Transactions on Reliability, vol. 61, no. 2, pp. 410-425, 2012.

[27] M. Compare and E. Zio, "Predictive maintenance by risk sensitive particle filtering," IEEE Transactions on Reliability, vol. 63, no. 1, pp. 134-143, 2014.

[28] H. Shi and J. Zeng, "Real-time prediction of remaining useful life and preventive opportunistic maintenance strategy for multi-component systems considering stochastic dependence," Computers \& Industrial Engineering, vol. 93, pp. 192-204, 2016.

[29] J. Li and Y. Ying, "A method to improve the robustness of gas turbine gas-path fault diagnosis against sensor faults," IEEE Transactions on Reliability, 2017.

[30] H. Mo, W. Wang, M. Xie, and J. Xiong, "Modeling and analysis of the reliability of digital networked control systems considering networked degradations," IEEE Transactions on Automation Science and Engineering, vol. 14, no. 3, pp. 1491-1503, 2017.

[31] B. Cai, Y. Liu, and M. Xie, "A dynamic-bayesian-network-based fault diagnosis methodology considering transient and intermittent faults," IEEE Transactions on Automation Science and Engineering, vol. 14, no. 1 , pp. 276-285, 2017

[32] H. Mo and M. Xie, "A dynamic approach to performance analysis and reliability improvement of control systems with degraded components," IEEE Transactions on Systems, Man, and Cybernetics: Systems, vol. 46, no. 10, pp. 1404-1414, 2016.

[33] A.-C. Romain and J. Nicolas, "Long term stability of metal oxidebased gas sensors for e-nose environmental applications: An overview," Sensors and Actuators B: Chemical, vol. 146, no. 2, pp. 502-506, 2010.

[34] A. Vergara, S. Vembu, T. Ayhan, M. A. Ryan, M. L. Homer, and R. Huerta, "Chemical gas sensor drift compensation using classifier ensembles," Sensors and Actuators B: Chemical, vol. 166, pp. 320-329, 2012.

[35] B. Pourbabaee, N. Meskin, and K. Khorasani, "Robust sensor fault detection and isolation of gas turbine engines subjected to time-varying parameter uncertainties," Mechanical Systems and Signal Processing, vol. 76, pp. 136-156, 2016

[36] R. Dorr, F. Kratz, J. Ragot, F. Loisy, and J.-L. Germain, "Detection, isolation, and identification of sensor faults in nuclear power plants," IEEE Transactions on Control Systems Technology, vol. 5, no. 1, pp. 42-60, 1997.

[37] Z.-S. Ye and M. Xie, "Stochastic modelling and analysis of degradation for highly reliable products," Applied Stochastic Models in Business and Industry, vol. 31, no. 1, pp. 16-32, 2015.

[38] Q. Zhai and Z.-S. Ye, "Rul prediction of deteriorating products using an adaptive wiener process model," IEEE Transactions on Industrial Informatics, vol. 13, no. 6, pp. 2911-2921, 2017.

[39] X.-L. Meng and D. B. Rubin, "Maximum likelihood estimation via the ecm algorithm: A general framework," Biometrika, vol. 80, no. 2, pp. 267-278, 1993.

[40] D. N. Politis and M. Sherman, "Moment estimation for statistics from marked point processes," Journal of the Royal Statistical Society: Series B (Statistical Methodology), vol. 63, no. 2, pp. 261-275, 2001.

[41] X. Zhao, B. Liu, and Y. Liu, "Reliability modeling and analysis of load-sharing systems with continuously degrading components," IEEE Transactions on Reliability, vol. 67, no. 3, pp. 1096-1110, 2018.

[42] B. Liu, R.-H. Yeh, M. Xie, and W. Kuo, "Maintenance scheduling for multicomponent systems with hidden failures," IEEE Transactions on Reliability, vol. 66, no. 4, pp. 1280-1292, 2017. 
[43] R. Mehra, "On the identification of variances and adaptive kalman filtering," IEEE Transactions on Automatic Control, vol. 15, no. 2, pp. 175-184, 1970

[44] X.-S. Si, W. Wang, C.-H. Hu, and D.-H. Zhou, "Estimating remaining useful life with three-source variability in degradation modeling," IEEE Transactions on Reliability, vol. 63, no. 1, pp. 167-190, 2014.

[45] E. Skordilis and R. Moghaddass, "A condition monitoring approach for real-time monitoring of degrading systems using kalman filter and logistic regression," International Journal of Production Research, vol. 55, no. 19, pp. 5579-5596, 2017.

[46] Y. Liu, "Adaptive just-in-time and relevant vector machine based softsensors with adaptive differential evolution algorithms for parameter optimization," Chemical Engineering Science, vol. 172, pp. 571-584, 2017.

[47] Y. Liu, J. Guo, Q. Wang, and D. Huang, "Prediction of filamentous sludge bulking using a state-based gaussian processes regression model," Scientific Reports, vol. 6, p. 31303, 2016.

[48] D. Wang, F. Yang, Y. Zhao, and K.-L. Tsui, "Prognostics of lithiumion batteries based on state space modeling with heterogeneous noise variances," Microelectronics Reliability, vol. 75, pp. 1-8, 2017.

\section{APPENDIX}

\section{A. Initial guess of the optimal maintenance decision}

A near-optimal initial guess is achieved by minimizing the long run maintenance cost rate under the condition of perfect inspection. Since a renewal cycle ends with either preventive replacement or corrective replacement, optimization of the long run cost rate can be expressed as

$$
\begin{aligned}
\min C^{(0) \infty}\left(\Delta T^{(0)}, R_{s}^{(0)}\right) & =\frac{c_{i} E\left[N_{I}^{(0)}\left(\mathrm{Z}^{(0)}\right)\right]+c_{p} E\left[1_{\{P R\}}\right]}{E\left[\mathrm{Z}^{(0)}\right]} \\
& +\frac{c_{c} E\left[1_{\{C R\}}\right]+c_{d} E\left[W^{(0)}\left(\mathrm{Z}^{(0)}\right)\right]}{E\left[\mathrm{Z}^{(0)}\right]}
\end{aligned}
$$

subject to $R_{S}^{(0)} \in(0,1)$

where $1_{\{C R\}}$ denotes the event that the renewal cycle ends with corrective replacement, and $1_{\{P R\}}$ stands for the event that the renewal cycle ends with preventive replacement. Note that we use the superscript (0) to distinguish from the imperfect inspection case. In the case where inspection can accurately observe the system state, due to the Markov property of Wiener process, the one-inspection-ahead reliability conditioned on the observation history $\mathbf{Y}_{1: k}^{(0)}$ is identical as that conditioned on the current system state $x_{k}$,

$$
R^{(0)}\left(t_{k}+\Delta T^{(0)} \mid \mathbf{Y}_{1: k}^{(0)}\right)=R^{(0)}\left(t_{k}+\Delta T^{(0)} \mid x_{k}\right)
$$

According to the independent increment property of Wiener process, the one-inspection-ahead conditional reliability can be obtained as

$$
\begin{array}{r}
R^{(0)}\left(t_{k}+\Delta T^{(0)} \mid x_{k}\right)=\Phi\left(\frac{\zeta-x_{k}-\lambda \Delta T^{(0)}}{\sigma \sqrt{\Delta T^{(0)}}}\right) \\
-\exp \left(\frac{2 \lambda\left(\zeta-x_{k}\right)}{\sigma^{2}}\right) \Phi\left(\frac{-\left(\zeta-x_{k}\right)-\lambda \Delta T^{(0)}}{\sigma \sqrt{\Delta T^{(0)}}}\right)
\end{array}
$$

It is clearly shown that $R^{(0)}\left(t_{k}+\Delta T \mid x_{k}\right)$ is continuous and shows a monotone decreasing trend with respect to the system state $x_{k}$. The problem of finding the reliability threshold $R_{S}^{(0)}$ is identical to obtaining the state threshold $M$ such that the system is preventively replaced when its state exceeds $M$. Optimization of (A1) equals to

$$
\begin{aligned}
& \min C^{(0) \infty}\left(\Delta T^{(0)}, M\right) \\
& \text { subject to } M \in(0, \zeta)
\end{aligned}
$$

Let $T_{M}$ be the first passage time to the state threshold $M, T_{M}=$ $\inf \{t: X(t) \geq M\}$. Based on how the regenerative process $\{X(t), t \geq 0\}$ ends in a renewal cycle, it can be classified into two types: ending with corrective replacement or preventive replacement.

At the $k$ th inspection, corrective replacement is performed if the system state exceeds the failure threshold $\zeta\left(X_{k}>\zeta\right)$ while it remains below the preventive replacement threshold $M$ at the previous inspection $\left(X_{k-1}<M\right)$. The probability for such an event is given as

$$
\begin{gathered}
P\left(X_{k}>\zeta \cap X_{k-1}<M\right)=\left(1-F_{T_{M}}\left((k-1) \Delta T^{(0)}\right)\right) . \\
\int_{0}^{M} F_{\zeta-x}\left(\Delta T^{(0)} ; x\right) f_{X}\left(x ;(k-1) \Delta T^{(0)}\right) d x
\end{gathered}
$$

where

$$
\begin{gathered}
f_{X}(x ; t)=\frac{1}{\sqrt{2 \pi \sigma^{2} t}} \exp \left(\frac{(x-\lambda t)^{2}}{2 \sigma^{2} t}\right) \\
F_{\zeta-x}(t ; x)=1-\Phi\left(\frac{\zeta-x-\lambda t}{\sigma \sqrt{t}}\right) \\
+\exp \left(\frac{2 \lambda(\zeta-x)}{\sigma^{2}}\right) \Phi\left(\frac{-(\zeta-x)-\lambda t}{\sigma \sqrt{t}}\right)
\end{gathered}
$$

$$
\begin{aligned}
& F_{T_{M}}(t)=P\left(T_{M}<t\right)=1-\Phi\left(\frac{M-\lambda t}{\sigma \sqrt{t}}\right) \\
& +\exp \left(\frac{2 \lambda M}{\sigma^{2}}\right) \Phi\left(\frac{-M-\lambda t}{\sigma \sqrt{t}}\right)
\end{aligned}
$$

Preventive replacement is carried out when the system state at inspection satisfies $\zeta>X_{k}>M$ and $X_{k-1}<M$. The associated probability is expressed as

$P\left(\zeta>X_{k}>M \cap X_{k-1}<M\right)=\left(1-F_{T_{M}}\left((k-1) \Delta T^{(0)}\right)\right)$
$\int_{0}^{M}\left(F_{M-x}\left(\Delta T^{(0)} ; x\right)-F_{\zeta-x}\left(\Delta T^{(0)} ; x\right)\right) f_{X}\left(x ;(k-1) \Delta T^{(0)}\right) d x$

where

$$
\begin{aligned}
& F_{M-x}(t ; x)=1-\Phi\left(\frac{M-x-\lambda t}{\sigma \sqrt{t}}\right) \\
& +\exp \left(\frac{2 \lambda(M-x)}{\sigma^{2}}\right) \Phi\left(\frac{-(M-x)-\lambda t}{\sigma \sqrt{t}}\right)
\end{aligned}
$$


The long run cost rate can be obtained as

$$
\begin{aligned}
& C^{(0) \infty}\left(\Delta T^{(0)}, M\right)=\frac{c_{i}}{\Delta T^{(0)}}+c_{d} \sum_{k=1}^{\infty} p_{c}(k) \\
& +\frac{c_{p} \sum_{k=1}^{\infty} p_{p}(k)+c_{c} \sum_{k=1}^{\infty} p_{c}(k)-c_{d} \sum_{k=1}^{\infty} p_{c}(k) \int_{(k-1) \Delta T^{(0)}}^{k \Delta T^{(0)}} t d F_{T}(t)}{\Delta T^{(0)} \sum_{k=1}^{\infty} k\left(p_{c}(k)+p_{p}(k)\right)}
\end{aligned}
$$

where $p_{c}(k)=P\left(X_{k}>\zeta \cap X_{k-1}<M\right)$, and $\quad p_{p}(k)=$ $P\left(\zeta>X_{k}>M \cap X_{k-1}<M\right)$. By minimizing (A7), the optimal maintenance decision is achieved as

$$
\left\{\Delta T^{(0) *}, M^{*}\right\}=\arg \min C^{(0) \infty}\left(\Delta T^{(0)}, M\right)
$$

The initial guess of the optimal decision is given as $\left\{\Delta T^{(0) *}, R_{s}^{(0)^{*}}\right\}$, where

$$
\begin{aligned}
& R_{s}^{(0) *}=\Phi\left(\frac{\zeta-M^{*}-\lambda \Delta T}{\sigma \sqrt{\Delta T}}\right) \\
& -\exp \left(\frac{2 \lambda\left(\zeta-M^{*}\right)}{\sigma^{2}}\right) \Phi\left(\frac{-\left(\zeta-M^{*}\right)-\lambda \Delta T}{\sigma \sqrt{\Delta T}}\right)
\end{aligned}
$$

\section{B. State estimation with unknown sensor degradation rate}

For the case where the sensor degradation rate $\eta$ is unknown, but follows a Gaussian distribution, the state-space equation can be obtained as

$$
\left\{\begin{array}{l}
x_{k}=x_{k-1}+\lambda\left(t_{k}-t_{k-1}\right)+u_{k} \\
\eta_{k}=\eta_{k-1} \\
s_{k}=s_{k-1}+\eta_{k-1}\left(t_{k}-t_{k-1}\right)+v_{k} \\
y_{k}=x_{k}+s_{k}+\varepsilon_{k}
\end{array}\right.
$$

which can be rewritten as

$$
\left\{\begin{array}{l}
\mathbf{z}_{k}=\mathbf{A}_{k} z_{k-1}+\mathbf{B}_{k}+\mathbf{w}_{k} \\
y_{k}=\mathbf{H z}_{k}+\varepsilon_{k}
\end{array}\right.
$$

where

$$
\begin{gathered}
\mathbf{A}_{k}=\left[\begin{array}{ccc}
1 & 0 & 0 \\
0 & 1 & 0 \\
0 & t_{k}-t_{k-1} & 1
\end{array}\right], \mathbf{B}_{k}=\left[\begin{array}{c}
\lambda\left(t_{k}-t_{k-1}\right) \\
0 \\
0
\end{array}\right], \\
\mathbf{z}_{k}=\left[\begin{array}{c}
x_{k} \\
\eta_{k} \\
s_{k}
\end{array}\right], \mathbf{H}=\left[\begin{array}{lll}
1 & 0 & 1
\end{array}\right]
\end{gathered}
$$

$\mathbf{w}_{k} \in R^{3 \times 1}$ follows a 3-variante Gaussian distribution, $\mathbf{w}_{k} \sim$ $N\left(0, \mathbf{Q}_{k}\right)$, where

$$
\mathbf{Q}_{k}=\left[\begin{array}{ccc}
\sigma^{2}\left(t_{k}-t_{k-1}\right) & 0 & 0 \\
0 & 0 & 0 \\
0 & 0 & \delta^{2}\left(t_{k}-t_{k-1}\right)
\end{array}\right]
$$

The expectation and variance of $\mathbf{z}_{k}$ till the $k$ th inspection is given as

$$
\hat{\mathbf{z}}_{k \mid k}=\left[\begin{array}{c}
\hat{x}_{k \mid k} \\
\hat{\eta}_{k} \\
\hat{s}_{k \mid k}
\end{array}\right]=E\left(\mathbf{z}_{k} \mid \mathbf{Y}_{1: k}\right)
$$

$$
\mathbf{P}_{k \mid k}=\left[\begin{array}{ccc}
\chi_{x, k}^{2} & \chi_{x \eta, k}^{2} & \chi_{x s, k}^{2} \\
\chi_{x \eta, k}^{2} & \chi_{\eta, k}^{2} & \chi_{\eta s, k}^{2} \\
\chi_{x s, k}^{2} & \chi_{\eta s, k}^{2} & \chi_{s, k}^{2}
\end{array}\right]=\operatorname{cov}\left(\mathbf{z}_{k} \mid \mathbf{Y}_{1: k}\right)
$$

where $\chi_{x, k}^{2}=\operatorname{var}\left(x_{k} \mid \mathbf{Y}_{1: k}\right), \quad \chi_{\eta, k}^{2}=\operatorname{var}\left(\eta_{k} \mid \mathbf{Y}_{1: k}\right), \quad \chi_{s, k}^{2}=$ $\operatorname{var}\left(s_{k} \mid \mathbf{Y}_{1: k}\right), \quad \chi_{x \eta, k}^{2}=\operatorname{cov}\left(x_{k} \eta_{k} \mid \mathbf{Y}_{1: k}\right), \chi_{x s, k}^{2}=\operatorname{cov}\left(x_{k} s_{k} \mid \mathbf{Y}_{1: k}\right)$, $\chi_{\eta s, k}^{2}=\operatorname{cov}\left(\eta_{k} s_{k} \mid \mathbf{Y}_{1: k}\right)$. Similarly, the one-step-ahead prediction is expressed as

$$
\hat{\mathbf{z}}_{k \mid k-1}=\left[\begin{array}{c}
\hat{x}_{k \mid k-1} \\
\hat{\eta}_{k \mid k-1} \\
\hat{s}_{k \mid k-1}
\end{array}\right]=E\left(\mathbf{z}_{k} \mid \mathbf{Y}_{1: k-1}\right)
$$

$\mathbf{P}_{k \mid k-1}=\left[\begin{array}{ccc}\chi_{x, k \mid k-1}^{2} & \chi_{x \eta, k \mid k-1}^{2} & \chi_{x s, k \mid k-1}^{2} \\ \chi_{x \eta, k \mid k-1}^{2} & \chi_{\eta, k \mid k-1}^{2} & \chi_{\eta s, k \mid k-1}^{2} \\ \chi_{x s, k \mid k-1}^{2} & \chi_{\eta s, k \mid k-1}^{2} & \chi_{s, k \mid k-1}^{2}\end{array}\right]=\operatorname{cov}\left(\mathbf{z}_{k} \mid \mathbf{Y}_{1: k-1}\right)$

Estimation and update of the state and variance can be implemented as that in Section IV. The details are suppressed to avoid repetition. The initial expectation and variance is given as

$$
\hat{\mathbf{z}}_{0 \mid 0}=\left[\begin{array}{l}
0 \\
\mu_{\eta} \\
0
\end{array}\right], \mathbf{P}_{k \mid k}=\left[\begin{array}{ccc}
0 & 0 & 0 \\
0 & \sigma_{\eta}^{2} & 0 \\
0 & 0 & 0
\end{array}\right]
$$

\section{State estimation with dependent system and sensor degra- dation}

With the assumption that the system degradation has an additive impact on the sensor degradation, the state-space equation is given as

$$
\left\{\begin{array}{l}
x_{k}=x_{k-1}+\lambda\left(t_{k}-t_{k-1}\right)+u_{k} \\
s_{k}=s_{k-1}+\eta_{k-1}\left(t_{k}-t_{k-1}\right)+\alpha x_{k}+v_{k} \\
y_{k}=x_{k}+s_{k}+\varepsilon_{k}
\end{array}\right.
$$

where $\alpha$ is the parameter scaling the influence of system degradation on the sensor degradation. Similarly, the statespace equation can be rewritten as

$$
\left\{\begin{array}{l}
\mathbf{z}_{k}=\mathbf{A}_{k} z_{k-1}+\mathbf{B}_{k}+\mathbf{w}_{k} \\
y_{k}=\mathbf{H z}_{k}+\varepsilon_{k}
\end{array}\right.
$$

where

$$
\begin{aligned}
& \mathbf{A}=\left[\begin{array}{cc}
1 & 0 \\
\alpha & 1
\end{array}\right], \mathbf{B}_{k}=\left[\begin{array}{c}
\lambda\left(t_{k}-t_{k-1}\right) \\
\eta\left(t_{k}-t_{k-1}\right)+\alpha \lambda\left(t_{k}-t_{k-1}\right)
\end{array}\right] \\
& \mathbf{H}=\left[\begin{array}{ll}
1 & 1
\end{array}\right], \mathbf{w}_{k} \in R^{2 \times 1}, \mathbf{w}_{k} \sim N\left(0, \mathbf{Q}_{k}\right) \\
& \mathbf{Q}_{k}=\left[\begin{array}{cc}
\sigma^{2}\left(t_{k}-t_{k-1}\right) & 0 \\
0 & \delta^{2}\left(t_{k}-t_{k-1}\right)+\alpha^{2} \sigma^{2}\left(t_{k}-t_{k-1}\right)
\end{array}\right]
\end{aligned}
$$

With the above expressions, Kalman filter can be employed to estimate the system and sensor state. The details are similar to those in Section IV. 


\section{Impact of sensor failure}

The previous analysis assumes that the dedicated sensor is replaced together with the system and no sensor failure is taken into account. In this section we will investigate the impact of sensor failures on the maintenance actions. The sensor is replaced when it is found failed or along with system replacement. Note that since a sensor is usually far cheaper than the system, we do not incorporate the cost of sensor replacement in the evaluation of long run cost rate. When the system state is estimated by Kalman filter, we can have the conditional reliability at the next inspection as

$$
\begin{aligned}
R\left(t_{k}+\Delta T^{*} \mid \mathbf{Y}_{1: k}\right)= & \Phi\left(\frac{\zeta-\hat{x}_{k \mid k}-\lambda \Delta T^{*}}{\sqrt{\chi_{x, k}^{2}+\sigma^{2} \Delta T^{*}}}\right) \\
& -\exp \left(\frac{2 \lambda\left(\zeta-\hat{x}_{k \mid k}\right)}{\sigma^{2}}+\left(\frac{\sqrt{2} \lambda \chi_{x, k}}{\sigma^{2}}\right)^{2}\right) . \\
& \Phi\left(\frac{-\zeta+\hat{x}_{k \mid k}-\lambda \Delta T^{*}-2 \lambda \frac{\chi_{x, k}^{2}}{\sigma^{2}}}{\sqrt{\chi_{x, k}^{2}+\sigma^{2} \Delta T^{*}}}\right)
\end{aligned}
$$

Let $\pi_{k} \in\{0,1,2\}$ be the maintenance actions at the $k$ th inspection: $\pi_{k}=2$ stands for corrective replacement, $\pi_{k}=1$ indicates preventive replacement and $\pi_{k}=0$ represents do nothing. If the sensor functions at the $k$ th inspection, we have

$$
\pi_{k}=\left\{\begin{array}{l}
2, \text { if system fails } \\
1, \text { if } R\left(t_{k}+\Delta T^{*} \mid \mathbf{Y}_{1: k}\right)<R_{s}^{*} \\
0, \text { otherwise }
\end{array}\right.
$$

However, if the sensor fails at inspection, the system state cannot be detected and the maintenance decision has to be made based on the previous inspection information. It follows

$\pi_{k}=\left\{\begin{array}{l}2, \text { if system fails \& } R\left(t_{k-1}+2 \Delta T^{*} \mid \mathbf{Y}_{1: k-1}\right)<R_{s}^{*} \\ 1, \text { if system functions \& } R\left(t_{k-1}+2 \Delta T^{*} \mid \mathbf{Y}_{1: k-1}\right)<R_{s}^{*} \\ 0, \text { otherwise }\end{array}\right.$

where $R\left(t_{k-1}+2 \Delta T^{*} \mid \mathbf{Y}_{1: k-1}\right)$ is the two-inspections-ahead conditional reliability at the $(k-1)$ th inspection,

$$
\begin{aligned}
& R\left(t_{k-1}+2 \Delta T^{*} \mid \mathbf{Y}_{1: k-1}\right)=\Phi\left(\frac{\zeta-\hat{x}_{k-1 \mid k-1}-2 \lambda \Delta T^{*}}{\sqrt{\chi_{x, k-1}^{2}+2 \sigma^{2} \Delta T^{*}}}\right) \\
& -\exp \left(\frac{2 \lambda\left(\zeta-\hat{x}_{k-1 \mid k-1}\right)}{\sigma^{2}}+\left(\frac{\sqrt{2} \lambda \chi_{x, k-1}}{\sigma^{2}}\right)^{2}\right) . \\
& \Phi\left(\frac{-\zeta+\hat{x}_{k-1 \mid k-1}-\lambda 2 \Delta T^{*}-2 \lambda \frac{\chi_{x, k-1}^{2}}{\sigma^{2}}}{\sqrt{\chi_{x, k-1}^{2}+\sigma^{2} \Delta T^{*}}}\right)
\end{aligned}
$$

\section{E. Estimation of sensor degradation parameters}

The degradation parameters of the sensor can be estimated by maximum likelihood estimation (MLE). Let $Q(t)$ denote the measurements of the new sensor, $Q(t)=S(t)+\varpi$, where $\varpi$ is the measurement noise of the new sensor, following a Gaussian distribution, $\varpi \sim N\left(0, \vartheta^{2}\right)$. With the sensor degradation process of (2), the parameters under estimation are $(\eta, \delta, \vartheta)$. For notational convenience, let $\theta$ be the collection of the parameters under estimation, $\theta=(\eta, \delta, \vartheta)$. Since the sensor suffers a Wiener degradation process, to take advantage of the identical independent increment property of Wiener process, we will use the degradation increments to estimate the parameters. The sensor is inspected at time $\left\{t_{j}, j=1,2,3, \ldots, n\right\}$ and the associated measurements are denoted as $\left\{Q\left(t_{j}\right), j=1,2, \ldots, n\right\}$. Denote $\Delta t_{j}=t_{j}-t_{j-1}$ as the inspection intervals and $\kappa_{j}$ as the measurement increments, $\kappa_{j}=Q\left(t_{j}\right)-Q\left(t_{j-1}\right)$. It can be obtained that the set of measurement increments, $\kappa=$ $\left(\kappa_{1}, \kappa_{2}, \ldots, \kappa_{n}\right)$, follow a multivariate Gaussian distribution,

$$
\kappa \sim N(\eta \Delta t, \Sigma)
$$

where $\Delta t=\left(\Delta t_{1}, \Delta t_{2}, \ldots, \Delta t_{n}\right)$, and $\Sigma$ is the variance-covariance matrix, denoted as

$$
\Sigma_{j, k}=\operatorname{cov}\left(\kappa_{j}, \kappa_{k} \mid \theta\right)= \begin{cases}\delta^{2} \Delta t_{j}+\vartheta^{2}, & j=k=1 \\ \delta^{2} \Delta t_{j}+2 \vartheta^{2}, & j=k>1 \\ -\vartheta^{2}, & |j-k|=1 \\ 0, & \text { otherwise }\end{cases}
$$

Suppose that the degradation data can be collected from $N$ items. Let $i$ be the item index and $j$ the index of inspection epochs. For item $i$, the $j$ th inspection interval is denoted as $\Delta t_{i, j}=t_{i, j}-t_{i, j-1}$ and the $j$ th measurement increment is denoted as $\kappa_{i, j}=Q\left(t_{i, j}\right)-Q\left(t_{i, j-1}\right)$. Similarly, we can have $\kappa_{i}=\left(\kappa_{i, 1}, \kappa_{i, 2}, \ldots, \kappa_{i, n}\right)$ and $\Delta t_{i}=\left(\Delta t_{i, 1}, \Delta t_{i, 2}, \ldots, \Delta t_{i, n}\right)$. Since the degradation observations of each item follow a multivariate Gaussian distribution, given the sensor degradation data, we can have the log-likelihood function (up to a constant) as follows,

$$
l\left(\kappa_{1}, \kappa_{2}, \ldots, \kappa_{N}\right)=\sum_{i=1}^{N}\left(\ln \left|\Sigma_{i}\right|+\left(\kappa_{i}-\eta \Delta t_{i}\right)^{\prime} \Sigma_{i}^{-1}\left(\kappa_{i}-\eta \Delta t_{i}\right)\right)
$$

where $\Sigma_{i}$ is similarly defined as previous discussion. Estimates of $\theta$ can be obtained by maximizing the log-likelihood function $l\left(\theta \mid \kappa_{1}, \kappa_{2}, \ldots, \kappa_{N}\right)$.

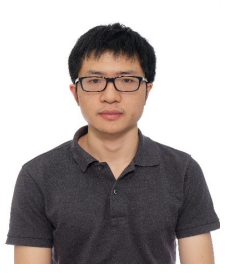

Bin Liu (M'17) is currently working as a lecturer in Department of Management Science, University of Strathclyde, Glasgow, UK. Before that he was working as a Postdoctoral Fellow in University of Waterloo, Canada. He received the B.S. degree in automation from Zhejiang University, China, in 2013, and the Ph.D. degree in industrial engineering from City University of Hong Kong, Hong Kong, in 2017. His research interests include risk analysis, reliability and maintenance modeling, decision making under uncertainty, and data analysis. 


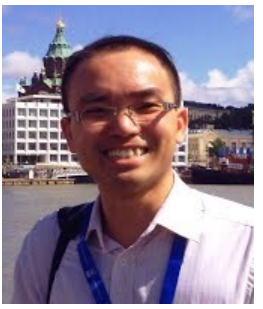

Phuc Do is currently working as an associate professor at Lorraine University, Research Centre for Automatic Control (CRAN CNRS UMR 7039), France. His research interests include stochastic modelling for reliability indicators prediction, maintenance policies and optimization, reliability importance measures and their related applications. He is co-guest editor of the Special Issue on Maintenance Modelling, Reliability Engineering System Safety, co-chair of the 9th and 10th International Conference on Modelling in Industrial Maintenance and Reliability (MIMAR9 and MIMAR10) and an IPC member of international conferences. He has published 60 research publications in international journals and conferences.

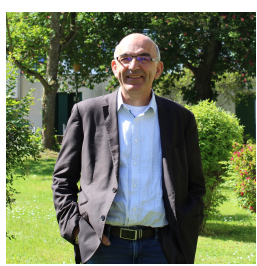

Benot Iung was born in 1962 in Nancy, France. $\mathrm{He}$ is full Professor of Prognostics and Health Management (PHM) at Lorraine University (France). He conducts research at the Nancy Research Centre for Automatic Control (CRAN, CNRS UMR 7039) where he is co-managing today a research group on Sustainable Industrial System Engineering (about 60 people). His research and teaching areas are related to dependability, advanced maintenance engineering, PHM and cyber-physical production system (CPP$\mathrm{S})$. In relation to these topics he took scientific responsibility for the participation of CRAN in a lot of national, European (i.e. REMAFEX, DYNAMITE) and international projects, for example, with China (i.e. EIAM-IPE, CENNET) and Chile (i.e. iMaPla). He has numerous collaborations with industry in the frame of Convention for Research Program (mainly in France with EDF, RENAULT) and serve as responsible of a common Lab called PHM-FACTORY with PREDICT SME (ANR LabCOM). $\mathrm{He}$ is the chairman of the IFAC TC5.1. (2017-2020). He was until 2014 the chairman of the IFAC WG A-MEST on advanced maintenance, and until 2018, the chairman of the ESRA TC on Manufacturing. He is a French CIRP fellow since 2017, a founding Fellow to the ISEAM and to the European IAM Academic and Research Network, a nominated member of the IFAC TC 5.3. He serves as correspondent on Factory of the Future for the University of Lorraine since 2016. Benot Iung has (co)-authored over 200 scientific papers and several books including the first e-maintenance book in Springer. He served as IPC member of various IEEE and IFAC conferences and developed expertise/reviewing for European Commission in the frame of H2020 from 2015. He is an Editor of the new IFAC Journal of System and Control. Benot IUNG received his B.S., M.S. and Ph.D. in Automatic Control, Manufacturing Engineering and Automation Engineering, respectively, from Lorraine University, and an accreditation to be research supervisor (2002) from this same University.

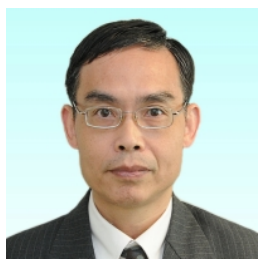

Min Xie (M'91-SM'94-F'06) received the Ph.D. degree in quality technology from Linkoping University, Linkoping, Sweden, in 1987. He is currently a Chair Professor with the City University of Hong Kong, Kowloon, Hong Kong. He has authored or coauthored numerous refereed journal papers, and some books on quality and reliability engineering, including Software Reliability Modeling (World Scientific, 1991), Weibull Models (Wiley, 2003), Computing Systems Reliability (Kluwer, 2004), and Advanced QFD Applications (ASQ Quality Press, 2003). Dr. Xie is the Department Editor of IIE Transactions and an Associate Editor of Reliability Engineering and System Safety. He was the recipient of the prestigious LKY Research Fellowship in 1991. He is on the editorial board of a number other international journals 